\title{
Assessment of Five Years' Comprehensive Management of HIV-Infected Children in Parakou
}

Agossou $\mathrm{J}^{1}$, Adédémy $\mathrm{JD}^{1}$, Noudamadjo $\mathrm{A}^{1}$, ${ }^{\text {, }}$, Sagbo $\mathrm{GG}^{2}$, Midété $\mathrm{JP}^{1}$, Lalya $\mathrm{F}^{2}$, Alao $\mathrm{MJ}^{2}$, d'Almeida $\mathrm{M}^{2}$, Séidou-Gounou-Bouraima $\mathrm{L}^{1}$, Avohou-Adjaho $\mathbf{P}^{1}$ and Adéothy-Koumakpai $\mathbf{S}^{2}$

${ }^{1}$ Mother \& Child Department, Faculty of Medecine, University of Parakou, Pediatric Unit of the Borgou Regional Hospital (CHD-B), Parakou, Benin ${ }^{2}$ Mother \& Child Department, Faculty of Health Sciences, University of Abomey-Calavi, HKM University Teaching Hospital (CNHU), Cotonou, Benin

\author{
Abstract \\ Introduction: The Pediatric Unit of the Borgou Regional Hospital (CHD-Borgou) in Parakou has initiated treating \\ HIV-infected children with Anti-Retroviral (ARV) drugs since 2005.
}

This study aimed to describe the profile and the future of the HIV-infected children who were provided medical care at the Pediatric Unit of the CHD-Borgou from 2005 to 2009.

Patients and methods: It is a retrospective study with descriptive and analytical objective, carried out from January 2005 to December 2009 on 105 HIV-infected children from both sexes cared in that Unit.

Results: HIV infection represented $0.43 \%$ of the children brought for medical examination during the study period. Among these children, $82.9 \%$ were at clinical stages 3 or 4 on inclusion into patient panel and $76.2 \%$ had severe immunodeficiciency. The proportion of children on Anti-Retroviral Therapy (ART) was $69.1 \%$.

The main opportunistic infections found were digestive (30.3\%), pulmonary $(28.5 \%)$, dermatological $(24.1 \%)$ and ENT (10.5\%) ones. The problems identified included lack of early diagnostic tools, frequent shortage of antiretroviral drugs, treatment or therapeutic failures, causes related to poor compliance and follow up. The lost of follow up rate was $39 \%$. Death rate was $33.3 \%$. Death was significantly associated with the advanced clinical stage of disease on admission $(p=0.002)$, with age at the time of screening $(p=0.017)$ and with follow up duration $(p=0.000)$.

Conclusion: Despite the difficulties noted, this study showed that, in limited resources settings like ours, it is possible to improve the clinical outcome of HIV infected children. The high mortality rate noted related to the delayed HIV diagnosis can be reduced by strengthening the Prevention of Mother to Child Transmission (PMTCT) program.

Keywords: HIV; ART; Children; Benin

\section{Introduction}

HIV infection remains a critical issue of public health and development, which reduces life expectancy in the African countries [1].

Sub-Saharan Africa is the most affected region in the world $[2,3]$. WHO estimates that about 2000 children are infected everyday globally, particularly in Sub-Saharan Africa [4]. Mother-To-Child Transmission (MTCT) of HIV is the most significant source of HIV infection in children $[1,3,5,6]$. Whereas in developed countries HIV pediatric infection is now under full control, in Africa, in adverse, high rates of births and mothers with HIV infection, difficult access to currently available interventions as well as expanded practice of extended breastfeeding imply a heavy burden of HIV in the child [6]. In fact, HIV infection is still one of the major causes of mortality and morbidity in the infant and child in Sub-Saharan Africa [2].

In Benin since 2002, HIV pandemic remains stationary with $1.2 \%$ prevalence without a significant variation from a year to another [7]. According to the national sentinel sero survey projections for the year 2013, the total amount of HIV infected patients is 68355 of which 10167 children under 15 years. The number of new HIV cases expected during 2013 is 2294 of among which 685 under 15 years with 594 annual children's death among infected ones. According to the same report the total number of children under ART is 3236 for a total need of $10167(31.8 \%)$ [8].

In the framework of the medical management of PLHIV, access to triple therapy begun in Benin in February 2002 on three sites, all located in Cotonou [9].
The CHD-Borgou pediatric unit has launched HIV management through ART since 2005. After five years of follow up of infected children in the unit, it would be convenient to review and take stock of the comprehensive management of the children followed.

\section{Study Site and Methods}

\section{Study site}

The study was carried out in the pediatric unit of CHD-Borgou in Parakou. This Pediatric Unit receives an average of 5000 patients per year, including 3500 hospitalizations. The pediatric unit works in close collaboration with the hospital laboratory and with the laboratory of the Borgou's Information, Prospective and Counseling Center (CIPEC) where CD4 counts as well as hematological, biochemical, serological and virological tests are executed.

The Borgou Regional Hospital in Parakou is a public tertiary

*Corresponding author: Alphonse Noudamadjo, Assistant Professor of Pediatrics, Medical Faculty of Parakou's University, Benin, 03, PO Box 09, Parakou, Benin, Tel: 0022990049007 or 0022994794149 ; E-mail: alphonse_ndama@yahoo.fr

Received September 05, 2013; Accepted October 29, 2013; Published November 02, 2013

Citation: Agossou J, Adédémy JD, Noudamadjo A, Sagbo GG, Midété JP, et al (2013) Assessment of Five Years' Comprehensive Management of HIV-Infected Children in Parakou. J AIDS Clin Res 4: 259. doi: 10.4172/2155-6113.1000259

Copyright: $\odot 2013$ Agossou J, et al. This is an open-access article distributed under the terms of the Creative Commons Attribution License, which permits unrestricted use, distribution, and reproduction in any medium, provided the original author and source are credited. 
care teaching hospital. It provides specialties as surgery and surgical specialties, medicine and medical specialties (psychiatry, dermatology, nephrology, and neurology), gynecology and obstetrics, pediatrics, radiology, laboratories, kinesi therapy and forensic medicine.

This pediatric ward is one of the main pediatric HIV management ward in the country and the most important in the northern part of the country. From 2005 to 2012, 710 children born from HIV positive mothers have been followed up through the PMTCT program.

Type and period of the study: It was a retrospective study with descriptive and analytical aims covering duration of 5 years from $1^{\text {st }}$ January, 2005 to 31 December, 2009.

Study population: It consists of children infected with HIV diagnosed on the basis of serological and /or clinical criteria.

Inclusion criteria: To be included into the study, children should have met three conditions as follows:

- Be under 15 years of age,

- Be detected as an HIV-infected individual,

- Be followed up at the pediatric unit.

The case recruitment was exhaustive. Every HIV positive children screened in the ward has been included.

Procedure: All the records of HIV-infected children followed are used to collect data recorded in a data processing sheet previously drafted.

Study variables: The variables studied were socio-demographic (age, gender, parents vital status, parents serological status), clinical (circumstances of diagnosis, morbidity in infected children, biological (CD4 count and hemoglobin concentration), therapeutic (children's proportion under ART, ART regimen, level of treatment adherence) and outcome (weight curve, CD4 count and Haemoglobin concentration evolution, opportunistic infections, ART failure, side effects).

\section{Definitions of some concepts and variables}

\section{HIV infection diagnosis: Were regarded as infected with HIV:}

- Children of 18 months to 15 years of age with HIV-serology positive status.

- Children under 18 months of age born from HIV-positive mothers showing at least one of the manifest clinical symptoms such as recurrent oral candidiasis, recurrent or severe pneumonia, persistent diarrhea, severe acute malnutrition and septicemia.

Virological diagnosis was not available at the CHD-Borgou during the period of study.

The age of children at diagnosis was considered as the one at admission in the follow-up program.

Diagnosis of pulmonary tuberculosis: The diagnosis of pulmonary tuberculosis was established on clinical and radiological arguments and favorable evolution under anti-tuberculosis treatment despite the negativity of tuberculin skin test and the negativity of the search for acid-alcohol resistant bacilli in sputum or gastric lavage fluid after Ziehl-Neelsen. No culture has been made.

Nutritional status: It was assessed using weight-for-height or upperarm circumference and/or presence of nutritional edema. Moderate
Acute Malnutrition (MAM) was defined by weight-for-height between -2 and -3 standard deviation and/or upper-arm circumference between 125 and $115 \mathrm{~mm}$. Severe Acute Malnutrition (SAM) was characterized by the presence of nutritional edema and/or a weight-for-height lower than $-3 \mathrm{ET}$ and/or a upper-arm circumference lower than $115 \mathrm{~mm}$.

Expression and interpretation of CD4: In children under 5 of age, CD4 were expressed in relative values in relation to the total of lymphocytes (\%). After five years, they were expressed in absolute values per $\mathrm{mm}^{3}$. The immunodeficiency was considered as very severe when CD4 ratio was lower than $15 \%$ or their number lower than $200 / \mathrm{mm}^{3}$. It was considered severe when CD4 ratio was between 15 and $20 \%$ or the number of CD4 between 200 and $350 / \mathrm{mm}^{3}$. The immunodeficiency was considered as moderate when CD4 ratio was between 20 and $25 \%$ or the number between 350 and $500 / \mathrm{mm}^{3}$. The absence of immunodeficiency was characterized by a CD 4 ratio higher than $25 \%$ or a number of CD 4 higher than or equal to $500 / \mathrm{mm}^{3}$.

Definition of anemia: Anemia was defined by a hemoglobin level lower than $11 \mathrm{~g} / \mathrm{dl}$. It was considered as severe if the hemoglobin level is lower than $7 \mathrm{~g} / \mathrm{dl}$.

\section{Definition of lost to follow up and irregular cases}

Irregular follow up: It involved children who started care in the pediatric unit and who had not been seen again at least at a check-up appointment made by the unit.

Lost to follow up: were considered as lost to follow up the children who begun care in the pediatric unit and who, at a given time, stopped following up during at least six months i.e. two appointments of three months missed.

Therapeutic compliance is defined as the degree of constancy and accuracy with which a patient follows a medical prescription [10] The data related to compliance with treatment were obtained from the children and/or of their parents' statements on the one hand, and through verification of follow up visits and ART renewal on the other hand. Non-compliance is considered in case of missing one follow up visit or one drug intake.

\section{Treatment failures}

Clinical failure is considered while facing: Recurrence of weight stagnation, adenopathies, a hepatosplenomegaly, recurrence of opportunistic diseases, and CD4 counts allowed confirming it.

Immunological failure criteria: Have been retained based on arguments as absence of increase of CD4 ratio (\% or absolute values) to ratios higher than the lower limit of the ratio recommended for the age after 24 weeks of treatment or return (\% or absolute rate) to a pretherapeutic CD4 ratio or below, in the absence of concurrent infections likely to explain CD4 decline or decline of CD4 ratio by more than $50 \%$ (absolute or \%) from optimum ratio under therapy in the absence of other infections likely to explain this decline [11].

Data collection and analysis; Data were collected by means of a data sheet drafted for this purpose. Then, they were captured and processed with SPSS 16.0 version software for Windows and Excel 2007 version. The qualitative variables were described in the form of rates and quantitative variables in the form of averages with standard deviation.

In order to know the factors associated with children death, a univariate analysis has been done using Epi-info 20016.04 version software. The association between variables was determined by crossing through Karl Pearson's Chi2 test at a 5\% significance threshold. 
Citation: Agossou J, Adédémy JD, Noudamadjo A, Sagbo GG, Midété JP, et al. (2013) Assessment of Five Years' Comprehensive Management of HIVInfected Children in Parakou. J AIDS Clin Res 4: 259. doi: 10.4172/2155-6113.1000259

Page 3 of 8

Ethical consideration: Local ethical committee agreement has been obtained. Confidentiality and anonymity were observed during data processing. In addition, the written informed consent of the parents for the use of their children's files was obtained.

\section{Results}

\section{Frequency of HIV infection cases in the pediatric unit}

During the time period of the study, 105 children with HIV infection were admitted in the CHD-Borgou pediatric unit. They accounted for $0.43 \%$ of the children seen at examination by medical professionals in the same period. Only HIV1 was found in all the infected children.

\section{Socio-demographic characteristics of the HIV-infected children and their parents}

Among the 105 children of this study the sex ratio was 1.28 . The mean average age at admission was $40.67 \pm 35$ months. The minimal age was 1 month and the maximal was 180 months. The median was 27 months.

The infected children were orphans in $38.1 \%$ of cases, including $20 \%$ of paternal ones, $13.3 \%$ of maternal ones and $4.8 \%$ of double orphans.

Their mothers were housewives or resellers in $70.3 \%$ and the fathers as civil servants in $32.7 \%$, followed by transporters or car drivers in $22.7 \%$ of cases.

Table 1 shows distribution of the socio-demographic characteristics of the children.

\section{Clinical characteristics of the HIV-infected children}

Among the fathers of the infected children, $60 \%$ knew the serologic status of their children.

In Table 2 are shown the characteristics related to the circumstances of disclosure of HIV infection in the children and their clinical stages according to WHO.

\begin{tabular}{|c|c|c|}
\hline & Number & Percentage (\%) \\
\hline \multicolumn{3}{|c|}{ Age at the time of screening (month) $(\mathrm{N}=105)$} \\
\hline$[0-18]$ & 23 & 21.9 \\
\hline$[18-60]$ & 56 & 53.3 \\
\hline$[60-120]$ & 21 & 20.0 \\
\hline [120-180] & 5 & 4.8 \\
\hline \multicolumn{3}{|l|}{ Gender } \\
\hline Male & 59 & $56.2 \%$ \\
\hline Female & 46 & 43.8 \\
\hline \multicolumn{3}{|c|}{ Person taking care of the child $(\mathrm{N}=105)$} \\
\hline Father and mother & 57 & 54.3 \\
\hline Mother only & 27 & 25.7 \\
\hline Father only & 7 & 6.7 \\
\hline Grand-parents & 8 & 7.6 \\
\hline Guardian (male or female) & 6 & 5.7 \\
\hline \multicolumn{3}{|c|}{ Serologic status of parents $(\mathrm{N}=105)$} \\
\hline HIV-positive father & 33 & 31.7 \\
\hline HIV-negative father & 4 & 4.0 \\
\hline Father with unknown status & 68 & 64.4 \\
\hline HIV-positive mother & 85 & 81.2 \\
\hline HIV-negative mother & 0 & 0 \\
\hline Mother with unknown status & 20 & 18.8 \\
\hline
\end{tabular}

Table 1: Distribution of the HIV-infected children followed in the CHD-Borgou pediatric unit from 2005 to 2009 according to socio-demographic characteristics.
The Table 3 indicates the frequency of the diseases found in the followed children at the time of screening.

Among the digestive diseases counted, oral candidiasis and persistent diarrhea accounted for $64.2 \%$ and $35.8 \%$ respectively. In the category of pulmonary diseases, recurrent pneumonia represented $90.2 \%$ of cases and lung tuberculosis accounted for $9.8 \%$. As regards skin diseases, they included prurigo, superficial mycoses, impetigo, zona, Kaposi sarcoma and molluscum contagiosum respectively in $46 \%, 16.2 \%, 16.2 \%, 13.5 \%, 5.4 \%$ and $2.7 \%$ of cases.

\section{Initial biological characteristics of the HIV-infected children}

Among the 105 children followed, 84 had benefitted from CD4 counts at the time of screening. According to $\mathrm{CD} 4$ values, 64 children were suffering from severe deficiency $(76.2 \%), 6$ in advanced deficiency (7.1\%), 4 in moderate deficiency (4.8\%) and 10 without immunodeficiency (11.9\%). As regards hematological characteristics, $57.7 \%$ of the infected children had anemia, including $6.7 \%$ with severe anemia. The average level of hemoglobin before ART initiation was $10 \mathrm{~g} / \mathrm{dl}$.

\section{Therapeutic characteristics of the HIV-infected children}

Among the infected children, 67 had been put on ART (63.8\%). The rest includes the dead before ART induction ( 8 cases) and the lost to follow up before being put on ART (30 cases)

After screening, the mean time for ART induction was $60 \pm 45$ days.

The ART regime used consisted of AZT $+3 \mathrm{TC}+\mathrm{NVP}$ in $67.2 \%$ of cases, $\mathrm{AZT}+3 \mathrm{TC}+\mathrm{NFV}$ in $25.4 \%, \mathrm{D} 4 \mathrm{~T}+3 \mathrm{TC}+\mathrm{NVP}$ in $4.5 \%$, $\mathrm{AZT}+3 \mathrm{TC}+\mathrm{IDV}$ in $1.5 \%$ and $\mathrm{AZT}+3 \mathrm{TC}+\mathrm{EFV}$ in $1.5 \%$ of cases.

\section{Characteristics of the progression of HIV-infected children on ART}

The mean follow-up duration was $12.53 \pm 11$ months. Figures

\begin{tabular}{|c|c|c|}
\hline & Number & Percentage $(\%)$ \\
\hline \multicolumn{3}{|l|}{ Screening circumstances $(\mathrm{N}=105)$} \\
\hline Hospitalization & 70 & 66.7 \\
\hline Infected parents known & 13 & 12.4 \\
\hline PMTCT failure & 11 & 10.5 \\
\hline After the death of a parent & 8 & 7.6 \\
\hline HIV- positive screening among the siblings & 2 & 1.9 \\
\hline Randomized screening & 1 & 0.9 \\
\hline \multicolumn{3}{|l|}{ WHO clinical stages at screening $(\mathrm{N}=105)$} \\
\hline Stage 1 & 11 & 10.5 \\
\hline Stage 2 & 7 & 6.7 \\
\hline Stage 3 & 38 & 36.2 \\
\hline Stage 4 & 49 & 46.7 \\
\hline
\end{tabular}

Table 2: Distribution of the HIV-infected children followed in the CHD-Borgou pediatric unit from 2005 to 2009 according to the circumstances of HIV-positive status disclosure and $\mathrm{WHO}$ clinical stage at the time of screening.

\begin{tabular}{|l|c|c|}
\hline & Number of cases & Frequencies (\%) \\
\hline Digestive diseases & 53 & 50.5 \\
\hline Severe acute malnutrition & 41 & 39 \\
\hline Lung diseases & 41 & 39 \\
\hline Skin diseases & 37 & 35.2 \\
\hline ENT diseases & 15 & 14.3 \\
\hline Chronic lymphadenopathy & 13 & 12.4 \\
\hline Neurological diseases & 8 & 7.6 \\
\hline
\end{tabular}

Table 3: Frequency of diseases found in the HIV infected children followed in the CHD-Borgou pediatric unit at the time of screening. 
1 and 2 show average weight progression, CD4 average values and hemoglobin level of the children on ART.

Regarding the progression trends and regularity of the HIVinfected children on ART followed at the pediatric unit, it was noted that $44(41.9 \%)$ were regular, $14(13.3 \%)$ irregular, $41(39 \%)$ lost to follow up, 23 (21.9\%) dead including 17 (25.37\%) on ART and 6 (5.7\%) transferred to another care site. The mean follow-up duration of dead children on ART was 4.64 months, with a range from 1 to 28 months and a median of 2 months. Of the 17 died children who died on ART, $8(47 \%)$ were in the first month of treatment the mean age at the death was $34.91 \pm 33$ months.

The deaths registered were significantly associated with the WHO advanced clinical stages $(\mathrm{p}=0.002)$, with age at the time of screening $(\mathrm{p}=0.017)$ and with the follow up length of time $(\mathrm{p}=0.000)$.

Other features on the monitoring of the 67 children on ART were changing of ARV drugs in 21 cases (31\%), ARV drugs side effects in 20 cases (29.9\%), ARV drugs shortage, poor compliance with the ART respectively in 18 cases each $(26.9 \%)$ and treatment failure in 3 cases (4.5\%).

The ART related side effects were cutaneous rash in 9 cases $(13.4 \%)$ and transaminase elevation in 6 cases (8.9\%). Lyell syndrome, digestive intolerance, creatininemia elevation, hyper-amylasemia and severe anemia were respectively found in one case each (1.5\%).

During the follow up, 21 children (31.3\%) were hospitalized once, 5 children $(7.5 \%)$ twice and 2 children (3\%) three times. The reasons for hospitalization of these children are shown in Table 4.

\section{Discussion}

The study has described the comprehensive follow up of the children with HIV infection at the Borgou Regional Hospital.

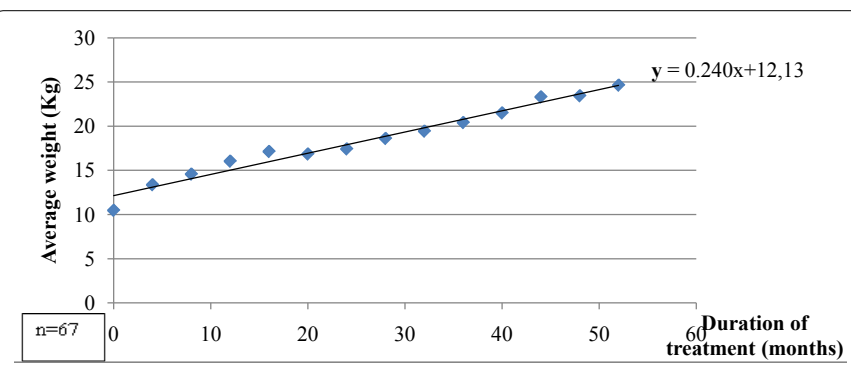

Figure 1: Progression of the average weight of the HIV-infected children on ART followed in the CHD-Borgou pediatric unit from 2005 to2009.

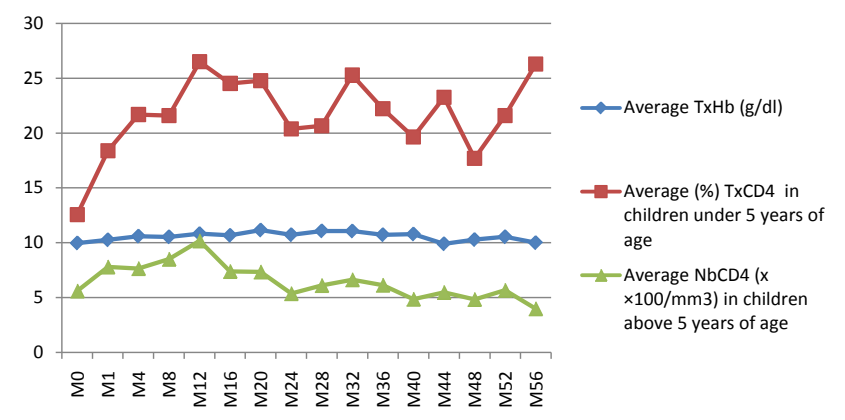

Figure 2: Progression of the CD4 average values and hemoglobin level of the HIV-infected children on ART followed at the CHD-Borgou pediatric unit from 2005 to 2009 .
It has allowed us to determine the place of HIV infection among the diseases encountered in the pediatric unit and to describe the clinical, biological profile and progression of the followed children.

These study limitations are linked to its retrospective nature, the technical laboratory level and the hospital part of its implementation.

As our study was conducted only at the pediatric unit of the Borgou Regional Hospital, its findings should not be considered as likely to be superposed on the national health situation. As it is usual in any retrospective study, data collection was not complete due to missing informations in some medical folders. However, this has no negative impact on the quality of the results. The exhaustive sampling and statistical tests used have enabled us to minimize possible biases.

On the second way, the technical level of the laboratory didn't allow the research team to perform consistent laboratory tests as PCR or viral load which are important in the follow up of children living with HIV. Thirdly, the number of loss of follow up needs to be investigated in another study to dig out the main reasons of this loss in order to improve their adherence to the program (PMTCT and HIV/ AIDS management program). Although we cannot extrapolate on the results found in this work, it brings out some important considerations regarding HIV patients follow up and management.

\section{Place of HIV infection in child at CHD-Borgou}

This relatively low rate of HIV infected children indicates a real underrating of the importance of this infection in hospital, for no systematic HIV serological test is done on all the children hospitalized, much less on all the children seen at medical examination.

Moreover, all the results of requested serological tests during the study period were not obtained due to repeated shortages of reagents at laboratory.

Eventually, the unavailability of tools for early diagnosis before 18 months age of the HIV infection in the children exposed to HIV (children born from HIV-positive mothers) could also explain this low rate. In our context, this implies a sub-screening of HIV infection at the hospitals.

This rate is identical with the one found by Maneffo [12] at Cotonou in 1997 (0.44\%) and by Belemou [13] in Bamako in 2002 (0.3\%).

\section{Socio-demographic characteristics of the infected children at the time of screening}

The mean age of the children on inclusion in this study is lower than the one found by Koueta et al. [14]; in 2008 at the Teaching Hospital (CHU) of Ouagadougou i.e. 48 months. The medium age was 27 months, which is lower than the one found by Chauhan et al. [15]; in 2010 in India i.e. 60 months.

The age group most affected in our study was the one from 18 months to 5 years $(53.33 \%)$. Baleng Maah [16], noted the same in Bamako in 2005.

\begin{tabular}{|l|c|c|}
\hline & Number & Percentage (\%) \\
\hline Recurrent pneumonias & 8 & 11.9 \\
\hline Severe acute malnutrition & 8 & 11.9 \\
\hline Pulmonary tuberculosis & 5 & 7.5 \\
\hline Severe malaria & 4 & 6 \\
\hline Septicemia & 1 & 1.5 \\
\hline Severe anemia & 1 & 1.5 \\
\hline Lyell syndrome & 1 & 1.5 \\
\hline
\end{tabular}

Table 4: Distribution of children on ARV based on hospitalization reasons. 
The unavailability of early diagnosis equipment such as PCR before 18 months of age could justify the predominance of this age group in our study.

Children from 0 to 5 years represented $75.2 \%$ of the total number of children infected in our study. This outcome is comparable with the one Koumakpaï et al. [17] found in Cotonou in 1992 by reporting that $80 \%$ of the infected children showed symptoms before 3 years of age; this usually corresponds with the one of the clinical manifestations of vertical transmission of HIV infection.

Male frequency found in the study was similar to the one that Amidou [18] found in Cotonou in 2005 (56\%). This result is also identical with the one found by several authors: Chauhan et al. [15] in India in 2010, Komme [19] in Bamako in 2004, Mbika and Moyen [20] in Brazzaville in 1998 and Rouafi [21] in Bamako in 2005.

Almost half of the children (45.7\%) were not living with their two parents. This is due to the death of one of the parents or to the separation of the couple either because of AIDS or because of other social problems the couple had faced. So, the child burden became the responsibility of the unique parent or a guardian alive. It would be better if parents take care of their child, for the presence of both in the household provides the child with security and affection he needs for balanced development and formation of his personality.

About one child in three (33.3\%) has lost one parent and about 5\% were paternal and maternal orphans. These results are similar to the ones found by Amidou [18] in Cotonou in 2005; he noted that $32 \%$ of the orphans lost at least one parent and $5.4 \%$ of the orphans had lost both father and mother. Apart from HIV infection itself, the fact that one or both parents die from AIDS represents for the children not only a social vulnerability but also an important added medical vulnerability [22,23].

The majority of the mothers whose profession was specified were resellers or housewives $(70.3 \%)$ while about one father in three was a salaried employee.

Metsia Thiam [24] made the same remark in 2006 in Cotonou, noting that most mothers of infected children were exerting an activity in the informal sector or were housewives (82.08\%). According to the same author, salaried fathers constituted the majority $(60.37 \%)$. This major representation of the housewives or resellers could account for their weak economic power which would make them financially dependent. This situation could be the cause of their strong vulnerability. In addition, one notes a not less important percentage of fathers working as car drivers or transporters $(22.8 \%)$ who could have played a prominent role in the infection of the couple. Indeed, in 2012 in Benin, 3.2\% of drivers or transporters were infected with HIV [8].

However, Kra et al. [25] established in Côte d'Ivoire that HIV infection reaches any individual regardless of his academic level, religion and occupation.

Mother's serological status was known in $81.2 \%$ of cases against $35.7 \%$ as regards father. The fact that mothers are more ready than fathers to accept testing, explains this. Actually, the latter prefer to ignore their serological status and refuse screening. This behavior would be due to a denial of the disease or fathers would feel rather guilty, frustrated or ashamed.

All the mothers whose serological status is known were HIVpositive. Mother' seropositivity was therefore likely to be superposed on the one of the child. This constitutes an argument in support of predominant mother to fetal HIV transmission as several studies have demonstrated it, particularly in case of weakness of the PMTCT strategy $[1,13,20]$.

\section{Knowledge of the serologic status of the children infected by their fathers and their clinical characteristics}

It is established that the follow up of an infected child must take into account the child in a comprehensive way within the context of his family environment, involving the father in partnership with the mother, in order to ensure an integrated management of the family unit [2]. In this study, the disclosure of HIV seropositivity in children mostly happened during hospitalization for disease (66.7\%). This fact could be the outcome of the PMTCT strategy's weakness which is still a reality in Sub-Saharan Africa in general and in Benin particularly [4]. The infected child's inclusion into the cohort should be the result of the PMTCT failure if the latter were spread and well conducted.

Most of the study children were at clinic stages 3 or 4 at the time of screening. Actually, HIV infection was often discovered during an opportunistic infection. This situation delays ARV drugs therapy and both clinical and immunological improvement. Many studies have proved that an early management enables a rapid improvement in the infected children at clinical and immunological levels [15,26]. It is therefore necessary to improve the PMTCT strategy so as to include earlier children according to the new national recommendations [6]; the aim is the early start of ARV therapy in case of proven infection.

Concerning the main diseases encountered in the infected children at the time of screening, oral candidiasis was the most frequent among the digestive diseases. This remark is also obvious in the research works conducted by Amidou [18] and Amegatse [27] respectively in Cotonou in 2005 and in Lomé. Both authors had noted a predominance of oral candidiasis respectively in $68 \%$ and $57.40 \%$ of cases. According to a study conducted by Gnahore [28] in Côte d'Ivoire in 1990, oral candidiasis comes first among AIDS digestive symptoms in the SubSaharan Africa. These oropharyngeal candidiasis lesions are a leading cause for undernutrition in these children.

Regarding pulmonary infections, Ametgatse [27] and Amidou [18] also made the same remark with respective rates estimated at $61.3 \%$ and $20.48 \%$ for pneumonias caused by ordinary germs versus $13.3 \%$ and $5.5 \%$ for pulmonary tuberculosis. Moreover, the frequency of pulmonary tuberculosis found in this study is lower than the one noted by Chauhan et al. [15] in India in 2010, which was $36.1 \%$. The difficulties in tuberculosis diagnosis in the child in general and particularly in the child with HIV infection could explain this relatively poor frequency of tuberculosis in this study. Many authors [29-31] have reported this difficulty in the diagnosis of tuberculosis in the infected children.

Concerning prurigo, Samake [32] had found a similar percentage (41.5\%) in Mali. Regarding recurrent otitises our results are identical with the ones of Mutombo et al. [33]. In their study, they found a high frequency of suppurative otitis in the HIV-positive children.

Almost half of the study children had severe acute malnutrition.

As a matter of fact, HIV-infected run a high risk of exposure to malnutrition. In 1998 in Brazzaville, Mbika and Moyen found 60\% of wasting syndrome in the infected children [20]. In a study carried out in the same unit in 2010, Agossou et al. found 8.2\% of HIV infection in the children suffering from acute malnutrition [34]. The reasons put forward by some authors to establish a relationship between HIV infection and malnutrition are firstly, an insufficient intake of food 
further to anorexia associated to the disease, oral ulcerations or thrush. Secondly, it can be explained by an increased loss of nutrients due to malabsorption, diarrhea or HIV enteropathy. Thirdly, an increase in the basal metabolism further to opportunistic infections and to the HIV infection itself [2] can also be one of the explanations. Besides, it has been established that malnutrition-HIV infection co-morbidity has a pejorative prognostic value $[35,36]$.

\section{Initial biological characteristics of the infected children}

Considering the CD4, only 4 out of 5 children have had initial CD4 count. CD4 cells could have not been counted in the other children either because there were lots of follow up; they died just after HIV infection diagnosis or because of reagent shortage.

Out of the 84 children, 62 (76.2\%) had severe immunodeficiency. The fact that the children were tested at an advanced stage of the disease may explain this outcome.

This result is similar to the ones reached by Samake [32] in 2004 and Sangare [37] in 2003; both have reported respectively that $71.2 \%$ and $72.1 \%$ of the children had severe immunodeficiency on inclusion at the Gabriel Touré Teaching Hospital (CHU) of Bamako.

According to literature, progressive depletion of T CD4 lymphocytes which is a fundamental prognostic marker of the disease constitutes the main immuno-pathological symptoms induced by HIV infection. This absolute depletion in CD4 is closely associated with viral replication and connected with the progression of the disease [38].

More than half of the children studied (57.7\%) had biological anemia on inclusion. This result is close to the one achieved by Komme [19] in Mali in 2004, i.e. 58. 6\%. On the other hand, it is below the one found by Adéothy-Koumakpaï et al. [17] in Cotonou en 1992 (89\%), Baleng Maah [16] in Bamako in 2005 (85.7\%), Tanoh et al. [39] in Abidjan in 1994 (91\%) and Chauhan et al. in India in 2010 (69.14\%) [15]. Our results are identical with the ones of literature which reports that classically progression of HIV infection comes with progressive decline of hemoglobin level [40].

\section{Therapeutic characteristics of the children followed up}

The children who benefitted from introduction to antiretroviral therapy accounted for $69.1 \%$. The other children who had not been put on ARV therapy were either lost to follow up, dead or subject to their parents' refusal due to denial of the disease. This suggests active search for the lost to follow up and enhancement of therapeutic education. The ratio of children under ARV therapy in our study is lower than the one reported by Metsia Thiam [24] in Cotonou in 2006 i.e. 77.0\%.

This percentage of infected children on ARV in our study is greatly higher than to the one found by Boisseau et al. [41] in 2006 during a study carried out in 2004 on the difficulties of access to antiretrovirals in eighteen francophone Africa countries, including Benin. Actually, according to that study, only 0.1 to $9.6 \%$ of the PLHIV had access to ARV. Our results indicate progressive improvement of children's accessibility to ARV therapy in Benin.

In this study, the ARV combinations most used in first line were $\mathrm{AZT}+3 \mathrm{TC}+\mathrm{NVP}$ in $67.2 \%$ of cases, $\mathrm{AZT}+3 \mathrm{TC}+\mathrm{NFV}$ in $25.4 \%$ of cases, thus complying with the national recommendations [9]. In 2004 in Bamako, the most used protocols were $\mathrm{D} 4 \mathrm{~T}+3 \mathrm{TC}+\mathrm{NVP}$ in $25.7 \%$ of cases and AZT+3TC+NFV in $16.8 \%$ [32].

Difficulties had been noted during the follow up of the patients on ARV triple therapy. They were ARV shortage, ARV side-effects, poor compliance and treatment failure.
As far as ARV shortage is concerned, Nelfinavir shortage was the most experienced; it occurred in 2007 after the withdrawal of that molecule from the therapeutic arsenal at global level. That molecule has been replaced by Nevirapine which would cause the hypersensitivity reactions and biological disturbances noted. As HIV infection is a chronic disease, it is important to avoid shortages in the drug distribution network to guarantee to the patients a regular availability of ARV and better therapeutic efficiency.

Poor compliance or non-adherence was found in 18 children i.e. $26.1 \%$ against $36.36 \%$ in the cohort of Baleng Maah [16] in 2005 in Bamako.

The key factors associated with poor compliance have not been studied in our cohort. Nevertheless, according to Yankoty [42], the only factor significantly related to poor compliance was duration of treatment. In our work, the children who benefitted from a maximum 24-month follow up were more compliant.

As it is established that the therapeutic success of ARV triple therapy is ensured by compliance higher than 95\% [43], a good therapeutic education before initiation and along management would contribute to the improvement of compliance.

The three treatment failures noted have basically been clinical and immunological (as viral load or burden is not available). The secondline protocols used $(\mathrm{ABC}+\mathrm{DDI}+\mathrm{LPV} / \mathrm{r})$ complied with national recommendations [11]

\section{Evolutive characteristics of the children on ART}

The clinical and immunologic parameters of the children generally improved on ART.

As regards clinical characteristics, we noted good weight growth, which is what the upward linear trend of the average weight curve shows at different follow up periods.

Actually, about four out of five children (80.6\%) on ART were provided nutritional support through the assistance of the World Food Program (WFP). Moreover, the $39 \%$ of severe acute malnutrition cases of the cohort benefitted from nutritional rehabilitation by means of therapeutic foods.

With regard to hematological characteristics, the hemoglobin level remained around $10 \mathrm{~g} / \mathrm{dl}$ in the time despite the presence of AZT in most options used. This fact could be explained by the improvement of the nutritional status of these children on the one hand and by the decline of HIV's deleterious effect on the hematopoietic organs on the other hand.

As far as immunological characteristics are concerned, we also noted a satisfactory immunological progression both in children below 5 years of age and in those above 5 years (refer to Graph 2).

The overall progression revealed that $13.3 \%$ were irregular, $39 \%$ were lost to follow up and $21.9 \%$ have died. Among the children on ART, $21.9 \%$ benefitted from an irregular follow up; this raises compliance issue and therefore resistance risk; $21.9 \%$ were lost to follow up.

Delayed diagnosis could be the cause of high death rate in this cohort. In fact, all the dead children were admitted at WHO clinical stages 3 or $4(\mathrm{p}=0.002)$ or had severe immunosuppression in $79 \%$ of cases. In addition, death was premature for it was most noted during the first month of follow up $(\mathrm{p}=0.000)$. Those deaths occurred either in the pediatric unit or at home. Moreover, the deaths were most noted in 
Citation: Agossou J, Adédémy JD, Noudamadjo A, Sagbo GG, Midété JP, et al. (2013) Assessment of Five Years' Comprehensive Management of HIVInfected Children in Parakou. J AIDS Clin Res 4: 259. doi: 10.4172/2155-6113.1000259

the children under one year of age $(\mathrm{p}=0.017)$. These children constitute the first category of natural progression of HIV infection i.e. fast progressing elements who, having acquired infection in utero or during early perinatal period die before one year of age [2].

The death rate found in the study is similar to the one noted by Kam et al. [44] in 2001 in Ouagadougou (20\%) and Amegatse [27] in 2002 in Lomé (22.65\%).

The high rate of loss is a serious problem in the follow up of infected children. Most children lost to follow up (92.7\%) were children who had not been seen again just after screening $(\mathrm{p}=0.000)$. As reasons that may explain this situation, there is long time of serological results delivery, pre- and post-test counselling poorly carried out, socio-cultural real life experiences of the disease and fear of stigmatization. Furthermore, this situation can result from the fact that an important proportion of the followed children were orphans.

This early loss of child patients on the first medical examination was also noted by Lawson-Evi et al. [45] in 2010 in Togo.

\section{Conclusion}

This study has enabled to point out the difficulties associated with the management of HIV infected children at the CHD-Borgou in Parakou. It has also outlined the need to extend PMTCT to all the health facilities of the country. Moreover, it has recalled the urgency to improve the follow up of children born from HIV-positive mothers through early diagnosis using PCR and their putting on ART in case of PCR positivity. Eventually, it indicates that HIV screening should be systematically proposed in any sick child seen at medical examination or hospitalized in the pediatric unit after a well-informed counselling provided to the parents.

\section{References}

1. UNAIDS (2009) AIDS Epidemic Update. WHO/UNAIDS Report.

2. Tindyebwa D, Kayita J, Musoke J, Eley B, Nduati R, et al. (2006) Handbook on pediatric AIDS in Africa. ANECCA.

3. Yeni P (2008) Medical management of HIV-infected persons. Paris, MédecineSciences Flammarion.

4. UNAIDS (1999) Prevention of HIV Transmission from Mother to Child. Strategic Options. UNAIDS, Geneva.

5. Jackson H (2002) HIV Parent-Child Transmission, In: Jackson Helen (eds) AIDS in Africa: Continent in crisis. SAfAIDS 187-213.

6. Ministry of Public Health/National AIDS Control Program (PNLS) (2010) Policy, standards and procedures for the management of persons living with AIDS in BENIN.

7. National Institute of Statistics and Economic Analysis (INSAE) [Benin], Macro International Inc. (2012) Demographic and Health Survey-Benin (EDSB-IV) Benin 2011-2012. Claverton, Maryland, USA

8. Ministry of Public Health/National AIDS Control Program (PNLS) (2012) National Serosurvey of HIV and Syphilis in Sentinel sites in pregnant women.

9. UNAIDS/PNLS/ National AIDS Control Committee (CNLS) Benin (2010) UNGASS Country Progress Report. Libya.

10. Haynes RB, Taylor DW, Sackett DL (1979) Compliance in health care. Baltimore, Maryland. The John Hopkins University press.

11. Ministry of Health/PNLS/UNICEF/Benin (2007) Guide for the management of children HIVIAIDS exposed and infected in BENIN.

12. Maneffo Nafack EP (1997) Child HIV infection in Benin: epidemiological and clinical study at the University Teaching Hospital (CNHU) of Cotonou. Knowledge, attitudes and practices of child health services staff. Thesis Med, Cotonou 043.
13. Belemou B (2002) Pediatric AIDS' respiratory symptoms at CHU Gabriel Touré (Teaching hospital) about 141 cases. Thesis Med, Bamako 33.

14. Kouéta F, Yé D, Dao L, Zoungrana-Kaboré A, Ouédraogo SA, et al. (2008) [Lung disease and HIV infection in children at the Charles de Gaulle university pediatric hospital center in Ouagadougou (Burkina Faso)]. Sante 18: 15-18.

15. Chauhan NK, Mendiratta S, Mojumdar K, Madhu V (2010) Effect of mother to child transmission intervention strategies and clinical, hematological immunological characteristics in children born to HIV-1 infected mothers in India. J Ped Infect Dis 5: 49-56.

16. Baleng Maah B (2005) Antiretroviral's side-effects in children at the pediatric unit of the CHU Gabriel Touré (Teaching hospital) Thesis Med, Bamako.

17. Koumakpaï S, Dan V, Hazoume FA, Ayivi B (1992) Pediatric AIDS in Cotonou. Our experience related to 10 cases. Med Afr Noire 39: 510-513.

18. Amidou S (2000) Overview of opportunistic diseases in HIVIAIDS infected children from 0 to 15 years of age at CNHU-HKM Teaching Hospital of Cotonou. Thesis Med, Cotonou 1180.

19. Komme HC (2004) Progression of HIV seroprevalence at the pediatric unit of $\mathrm{CHU}$ Gabriel Touré Teaching Hospital from 1999 to 2002. Thesis Med Bamako 31.

20. Mbika C, Moyen G (1998) AIDS in pediatric intensive care. Med Afr Noire 45 29-32

21. Rouafi O (2005) Follow up of children born from HIV positive mothers at the pediatric unit of CHU Gabriel Touré Teaching Hospital: Outcomes of two years of activity. Thesis Med Bamako.

22. Crampin AC, Floyd S, Glynn JR, Madise N, Nyondo A, et al. (2003) The longterm impact of HIV and orphanhood on the mortality and physical well-being of children in rural Malawi. AIDS 17: 389-397.

23. Delfraissy Jean François (2002) Management of HIV-infected persons. Report, Paris: Flammarion Médecine Sciences 242-243.

24. Metsia Thiam M (2006) Management of HIV infection in children in CNHU/HKM of Cotonou. Thesis Med, Cotonou 1305

25. Kra O, Ouattara B, Ehui I, Tanon A, Doh M, et al. (2005) HIV infection seroprevalence in people attending the voluntary testing centre of Bouaké. Med Afr Noire 52: 419-425

26. Newell ML, Patel D, Goetghebuer T, Thorne C; European Collaborative Study (2006) CD4 cell response to antiretroviral therapy in children with vertically acquired HIV infection: is it associated with age at initiation? J Infect Dis 193 954-962.

27. Amegatse KA (2002) Opportunistic diseases in HIV positive children: study of 53 cases colligated at $\mathrm{CHU}$ Tokoin Teaching Hospital of Lomé from 1998 to 2000. Epidemiological and clinical aspects. Thesis Med, Lomé 11.

28. Gnahore D (1990) AIDS Oral symptoms in the Black African. Prospective study on 110 cases observed at the Teaching Hospital $(\mathrm{CHU})$ of Abidjan. Thesis Med Abidjan.

29. Kiwanuka J, Graham SM, Coulter JB, Gondwe JS, Chilewani N, et al. (2001) Diagnosis of pulmonary tuberculosis in children in an HIV-endemic area Malawi. Ann Trop Paediatr 21: 5-14

30. Madhi SA, Huebner RE, Doedens L, Aduc T, Wesley D, et al. (2000) HIV-1 coinfection in children hospitalised with tuberculosis in South Africa. Int J Tuberc Lung Dis 4: 448-454.

31. Madhi SA, Gray GE, Huebner RE, Sherman G, McKinnon D, et al. (1999) Correlation between CD4+ lymphocyte counts, concurrent antigen skin test and tuberculin skin test reactivity in human immunodeficiency virus type 1 in infected and uninfected children with tuberculosis. Pediatric infect Dis $\mathrm{J} 18$ : 800- 805

2. Samake M (2004) Child HIV infection: Clinical aspect and outcome of sixteen months of case management through antiretroviral drugs at the pediatric unit of the CHU Gabriel Touré Teaching Hospital. Thesis Med, Bamako.

33. Mutombo T, Keusse J, Sangare A (1996) AIDS and malnutrition in semi-rura areas' pediatric units: Dabou protestant hospital's experience in Côte d'Ivoire. Med Afr Noire 43: 72- 77

34. Agossou J, Adédémy JD, Noudamadjo A, Koukponou E, Adéothy-Koumakpa $\mathrm{S}$, et al. (2012) Acute malnutrition at hospital pediatric units in Parakou in the North-Bénin. Dakar Med 57: 86-94. 
Citation: Agossou J, Adédémy JD, Noudamadjo A, Sagbo GG, Midété JP, et al. (2013) Assessment of Five Years' Comprehensive Management of HIVInfected Children in Parakou. J AIDS Clin Res 4: 259. doi: 10.4172/2155-6113.1000259

35. Adedemy JD, Agossou J, Noudamadjo A, Koukponou E, Adeothy-Koumakpai $S$, et al. (2012) Evaluation of nutritional rehabilitation of acutely malnourished children at hospital pediatric units in Parakou. Dakar Med 57: 176-184

36. Dreyfuss ML, Fawzi WW (2002) Micronutrients and vertical transmission of HIV-1. Am J Clin Nutr 75: 959-970.

37. Sangare PO (2003) Child HIV infection: Clinical aspect and outcome of sixteen months of case management through antiretroviral drugs at the pediatric unit of the CHU Gabriel Touré Teaching Hospital. Doctoral Thesis in Medicine Bamako 78

38. Pantaleo G, Graziosi C, Demarest JF, Butini L, Montroni M, et al. (1993) HIV infection is active and progressive in lymphoid tissue during the clinically latent stage of disease. Nature 362: 355-358.

39. Tanoh FA, Timite KM, Camara R, Mahan L, Ekpini E, et al. (1994) Pneumopathy and AIDS. Pub Med Afr 130: 27-31.

40. Cassuto JP, Pesce A, Quaranta JF (1992) AIDS and HIV infection. Abrégés, 3ème édition.
41. Boisseau C, Degui H, Bruneton C, Rey JL (2006) [Poor access to antiretroviral treatment in French-speaking Africa: situation in 2004]. Med Trop (Mars) 66 589-592.

42. Yankoty LI (2008) Compliance with antiretroviral therapy in HIVIAIDS infected followed at the CNHU/HKM Teaching Hospital of Cotonou. Thesis Med Cotonou 1430

43. Mouala C, Kaba-Mebri J, Fikouma V, Wata JB, Gabrie P, et al. (2006) [Measuring treatment compliance in patients with HIV in Bangui, Central African Republic]. Med Trop (Mars) 66: 59-63.

44. Kam KL, Yé D, Ouedraogo S, Sanou I (2001) Opportunistic infections during HIV/ AIDS: Epidemiology and management. In Abstracts APANF 8th congress Libreville.

45. Lawson-Evi K, Mouhari-Toure A, Tchama R, Akakpo SA, Atakouma DY, et al. (2010) [Fate of children born to HIV positive mothers followed in the context of preventing mother-to-child transmission of HIV in Togo. Study of 1042 infants] Bull Soc Pathol Exot 103: 259-263. 